\title{
A Compact Soft X-Ray Spectrograph Combining High Efficiency and Resolution
}

\author{
R.R. Fäustlin ${ }^{a}$, U. Zastrau ${ }^{b}$, S. Toleikis ${ }^{a}$, I. Uschmann ${ }^{b}$, E. Förster ${ }^{b}$, \\ Th. Tschentscher ${ }^{* *}$ \\ ${ }^{a}$ Deutsches Elektronen-Synchrotron (DESY), \\ Notkestr. 85, 22607 Hamburg, Germany \\ ${ }^{b}$ Friedrich-Schiller-Universität Jena, \\ Max-Wien-Platz 1, 07743 Jena, Germany \\ ${ }^{c}$ European XFEL GmbH, \\ Albert-Einstein-Ring 19, 22761 Hamburg, Germany \\ E-mail: thomas.tschentscherexfel.eu
}

\begin{abstract}
A compact and light weight soft X-ray spectrograph covering 5-35 nm and employing a toroidal mirror and a variable line space reflection grating has been newly developed. Particular emphasis has been placed on achieving a large collection solid angle $\left(1.9 \times 10^{-3} \mathrm{sr}\right)$ and a high efficiency of the components in order to enable Thomson Scattering plasma diagnostics which has a small total cross section $\left(6.65 \times 10^{-25} \mathrm{~cm}^{2}\right)$. The instrument achieves a signal-to-noise ratio of 5 with a $13.5 \mathrm{~nm}$ source which isotropically emits $2.5 \times 10^{5}$ photons. A resolution $\lambda / \Delta \lambda=330$ was measured at $21 \mathrm{~nm}$ and the dispersion was calibrated. The instrument is housed inside a DN $100 \mathrm{CF}$ ultra high vacuum manipulator $\left(43 \times 46 \times 47 \mathrm{~cm}^{3}\right)$ which allows positioning relative to the source within $\pm 5 \mathrm{~mm}$ and $\pm 50 \mathrm{~mm}$ in $\mathrm{X}, \mathrm{Y}$ and $\mathrm{Z}$ direction, respectively. It can be used with or without entrance pinhole and is equipped with a motorized grating, a filter wheel with five filters, and a shutter. Altogether, these features make the spectrograph a versatile instrument which can be employed in a variety of physics applications such as line and bremsstrahlung spectroscopy or Thomson scattering.
\end{abstract}

KEYWORDS: soft x-ray spectroscopy; FEL radiation; synchrotron radiation; Thomson scattering; plasma diagnostic.

${ }^{*}$ Corresponding author. 


\section{Contents}

1. Introduction 1

2. Design 2

3. Measurements and Calibration 4

3.1 Dispersion 4

3.2 Resolution 5

3.3 Throughput 6

4. Integration into FLASH experiments 8

5. Conclusion 8

\section{Introduction}

Soft X-ray emission and scattering spectra are of great interest for various fields of physics. Possible applications range from the investigation of liquid and solid matter electronic structure [1] over lithography [2] and astrophysics [3, 4] to plasma diagnostics [5, 6]. In particular, plasma emission spectroscopy has profited from the abundance of emission lines in the range from 2 to $35 \mathrm{~nm}$ motivating the construction of advanced spectrographs [7, 8, 9]. The advent of soft X-ray free electron lasers (FELs) with the first of its kind in Hamburg (FLASH, DESY) [10] opens this spectral range for applications requiring higher intensity radiation. Here, we focus on the realization of soft X-ray Thomson scattering to investigate dense plasmas [11, 12, 13]. With this technique, temperature, density and degree of ionization of the plasma can be obtained via the spectral measurement of inelastic and elastic scattering components of the probe radiation [14]. It demands sufficient resolution to discriminate the different spectral contributions which is $\lambda / \Delta \lambda \geq 100$ in the FEL case [12]. More challenging, a high collection angle and efficiency of the spectrograph are required, as the total cross section for Thomson scattering is relatively small $\left(6.65 \times 10^{-25} \mathrm{~cm}^{2}\right)$. The greatest challenge is to integrate all these requirements into a most compact spectrograph so that it can be attached to various experimental chambers. Compactness and versatility are also what commercial products fail to offer. Therefore, the design of a new spectrograph was necessary. Beyond fulfilling these specifications, we aimed to construct a most versatile, easy to operate and in vacuum adjustable instrument with motorized filter change, motorized grating rotation and motorized shutter. In this paper we present the successful technical realization of these design goals as well as the calibration of the High Throughput, High Resolution Spectrograph for Soft $\underline{X}-$ Ray Light (HiTRaX) which has already been applied in various experiments [13, 15]. 


\section{Design}

For the use in soft $\mathrm{X}$-ray $(5-35 \mathrm{~nm})$ Thomson scattering the spectrograph requires a resolution $\lambda / \Delta \lambda \geq 100$ [12], a maximized efficiency due to the low cross section, various filters to suppress visible light over the whole spectral range, and a compact and light weight construction to be compatible with various experimental chambers and geometries. The combination of these design goals made the construction of a new instrument necessary. To increase the efficiency and in particular the acceptance angle, a collection and imaging optic is needed. As a dispersive element, we choose a planar variable line space (VLS) grating. It is more robust against the necessary evacuation and venting cycles of the instrument then the alternative transmission gratings, as the latter have to be free standing in this wavelength region (e.g. [9]). Also, larger transmission gratings are supported by a grid structure which in turn produces artifacts in the dispersion direction. Therefore, we chose customized reflection optics from Horiba Scientific ${ }^{\mathrm{TM}}$. These are platinum coated and consist of a toroidal mirror and a planar variable line space (VLS) holographic grating with an average line density of 800 lines $/ \mathrm{mm}$ (Figure 1 ). The mirror root mean square surface roughness was measured $0.6 \mathrm{~nm}$ which enables a high specular reflectivity throughout the wavelength range. Both optics have an active optical surface area of $50 \times 20 \mathrm{~mm}^{2}$. The mirror images the source with no magnification onto a Princeton Instruments PIXIS-XO: $2 \mathrm{~KB}^{\mathrm{TM}}$ back thinned CCD which has a pixel size of $13.5 \times 13.5 \mu \mathrm{m}^{2}$. The grating in between CCD and mirror disperses the image spectrally while the variable spacing of the lines focuses the light further along the dispersion direction improving spectral resolution and producing a flat focal plane. The CCD $(28 \times 7 \mathrm{~mm})$ then typically records a spectral bandwidth of $35 \mathrm{~nm}$ without moving grating or camera. To obtain high reflectivity even with soft X-ray light the optics are placed at grazing incidence geometry for total external reflection (78\% at 7.3 degree and $13.5 \mathrm{~nm}$ for the mirror [16]). The distance between mirror and source is $255 \mathrm{~mm}$ resulting in a solid collection angle of $1.9 \times 10^{-3} \mathrm{sr}$. Figure 1 shows the distances and angles of the optical components in the setup.

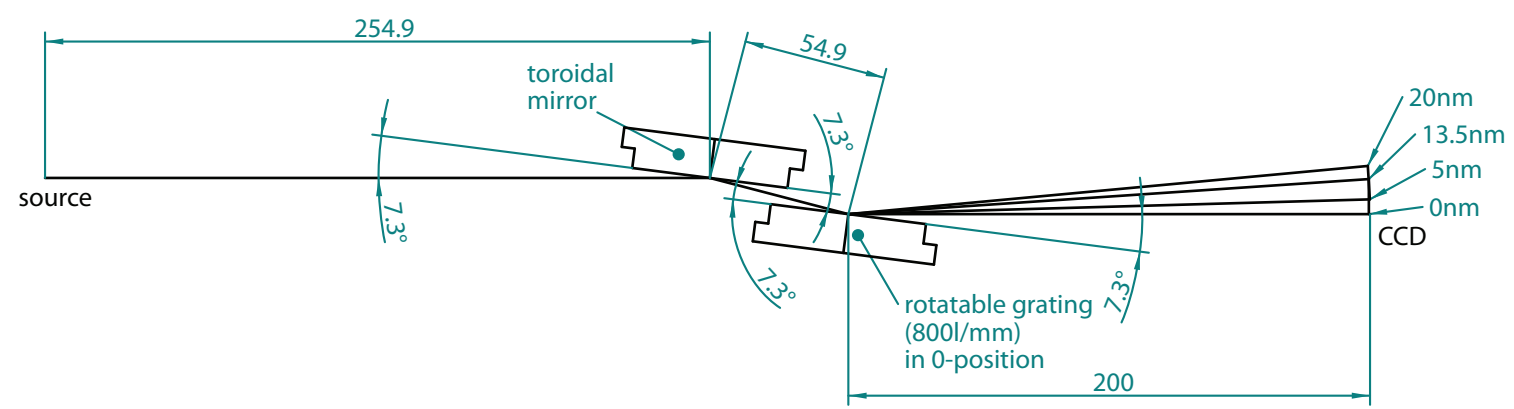

Figure 1. Geometry of the optics utilized in the spectrograph. Dimensions are in $\mathrm{mm}$ or degrees.

The spectrograph includes a motorized shutter to protect the optics from debris when not in use, motorized grating rotation with \pm 3 degree range of motion and a motorized filter wheel containing up to five filters (Figure 2). The electric motors are vacuum compatible and all are equipped with drive trains that do not require end switches, reducing possible sources of malfunction. The shutter is driven via a slipping clutch, the planar grating via an excenter wheel and the filter wheel can rotate perpetually. The filters help suppress visible light to which the CCD is sensitive as well 
as certain ranges in the soft X-ray spectrum depending on the filter material. The most used filters are $200 \mathrm{~nm}$ thick zirconium and aluminium which suppress visible light and have a bandpass of $6 \mathrm{~nm}-16 \mathrm{~nm}$ and $17.1 \mathrm{~nm}-75 \mathrm{~nm}$, respectively. These filters can be produced without fractures or holes and thus reduce visible and stray light reliably. Their dimensions $(28 \mathrm{~mm} \times 6 \mathrm{~mm})$ match the local soft X-ray beam size. The filter wheel is located between the CCD and the grating, away from the source, to protect fragile filter foils from debris that is common in laser plasma experiments. Furthermore, the filter wheel is surrounded by sufficient open space to minimize the pressure on the foils by air currents during evacuation and venting of the apparatus. The components facing the optical path have been anodized and dyed black to minimize stray light. Three apertures (Figure 2), shaped and experimentally adjusted to the optics outer dimensions also reduce stray light. The optics housing is built from aluminium to reduce weight to a minimum.
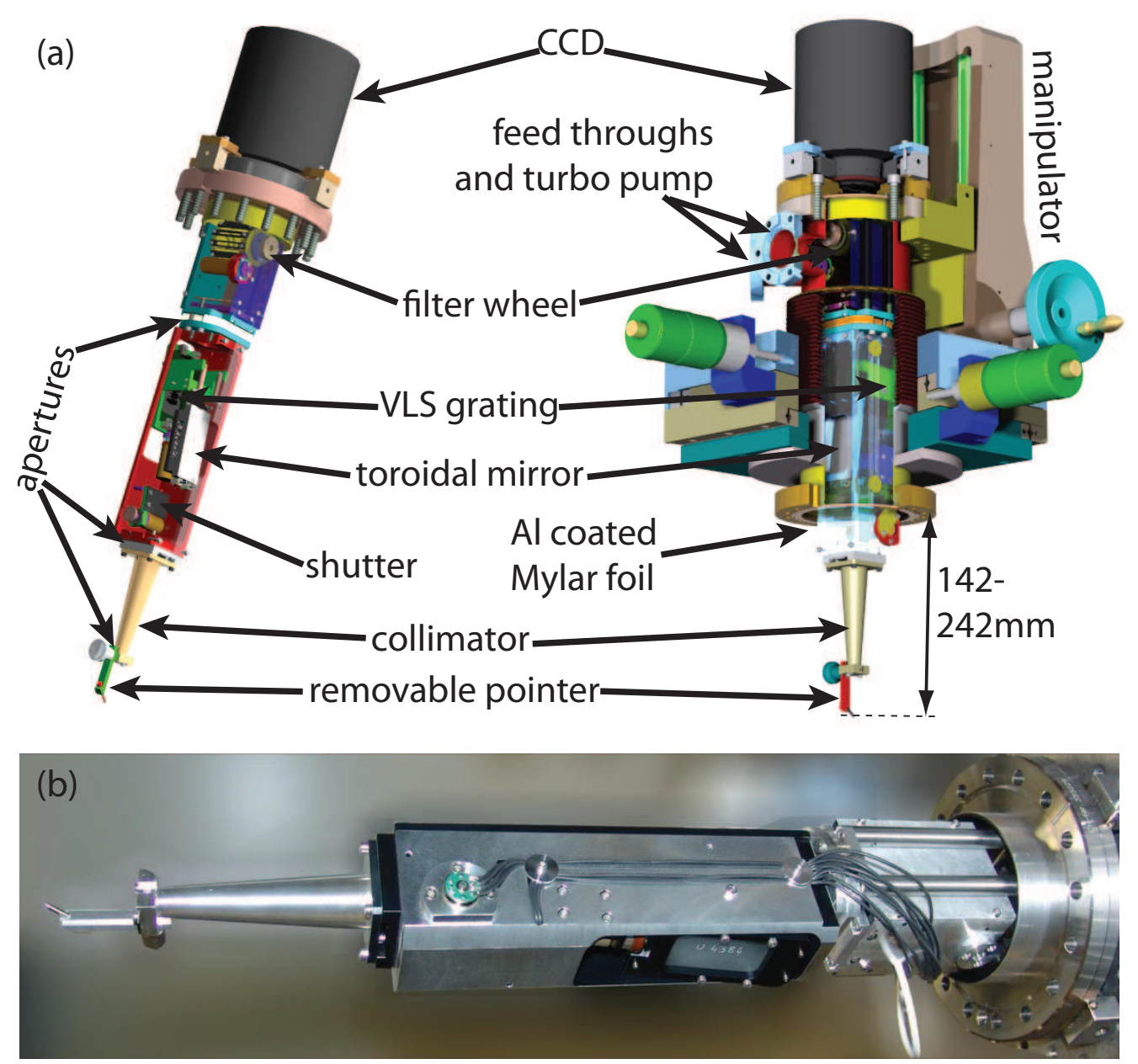

Figure 2. Three dimensional raytrace of the computer aided spectrograph design (a) indicating various components of the instrument in (right) and outside (right) of the manipulator. (b) Photograph of the spectrograph interior.

The whole spectrograph was built compact enough to be completely embedded in a DN $100 \mathrm{CF}$ ultra high vacuum three-way-manipulator with dimensions $43 \times 46 \times 47 \mathrm{~cm}^{3}$. Using the manipulator the instrument can be adjusted by $\pm 5 \mathrm{~mm}$ parallel and $\pm 50 \mathrm{~mm}$ perpendicular to the DN $100 \mathrm{CF}$ 
base flange. These degrees of freedom as well as a removable tip pointing to the source location reduce alignment time to the source significantly and allow the spectrograph to adapt to possibly changing source locations without having to break vacuum. The removable tip can be replaced with a pinhole holder so that high resolution spectra can be recorded even with large radiation sources. A flexible, $20 \mu \mathrm{m}$ thin Mylar foil coated on both sides with $250 \mathrm{~nm}$ of Al covers the gap between the spectrograph and the opening of the DN $100 \mathrm{CF}$ manipulator to prevent stray light from reaching the CCD while maintaining the full range of motion (Figure 2). The foil also separates the vacuum chamber of the experiment from the spectrograph interior, which is differentially pumped by means of a small turbo pump. Thus, the interior reaches an estimated pressure of $10^{-6} \mathrm{mbar}$. This prevents residual gas freeze-out on the CCD chip which is peltier cooled to $\sim 200 \mathrm{~K}$ with ambient air and no additional water cooling is necessary. The CCD housing is held in place using clamps. These press the CCD flange with an o-ring seal against the polished surface of the DN $100 \mathrm{CF}$. The construction allows the CCD to be rotated and shifted parallel to the flat flange surface when the instrument is not evacuated, thereby aligning the camera to the dispersion direction. Via this flange the filter wheel can be accessed quickly without having to remove the whole instrument and thus without loosing neither time nor alignment of the whole instrument. The above features allow a seamless integration and operation within the Thomson Scattering setup at FLASH for a most efficient use of experimental time.

\section{Measurements and Calibration}

\subsection{Dispersion}

The dispersion was calibrated using radiation from the DORIS-III synchrotron beam line BW3 [17, 18, 19] at DESY. The monochromator of this beam line delivers $10^{12}$ photons per second with an energy between $2 \mathrm{~nm}$ and $80 \mathrm{~nm}$ and a typical resolution of $\lambda / \Delta \lambda \cong 10^{4}$. A pinhole of $5 \mu \mathrm{m}$ diameter in the soft X-ray beam and at the source location of the spectrograph reduces intensity and produces a collimated ray of light entering the evacuated instrument $\left(10^{-6} \mathrm{mbar}\right)$ and generating a small image on the CCD. The location of the image in $0^{\text {th }}, 1^{\text {st }}$ and $2^{\text {nd }}$ order on the CCD is recorded for different wavelength settings of the beamline. Figure 3 shows this characterization of the spectrograph's dispersion.

Due to the geometry of the setup the dispersion is not perfectly but in in good approximation linear with $0.0170 \mathrm{~nm} /$ pixel $(1.259 \mathrm{~nm} / \mathrm{mm})$ at $13.5 \mathrm{~nm}$. The relative error of this linear fit is less than $1 \%$ in the wavelength region from $10.2 \mathrm{~nm}$ to $18.2 \mathrm{~nm}$ and below $0.1 \%$ within $12.3 \mathrm{~nm}$ to $14.8 \mathrm{~nm}$. Beyond this, the dispersion curve (Figure 3 ) can be fitted and interpolated with a parabola. Thus, radiation in the $1^{\text {st }}$ order and at a distance $x$ in pixels from the $0^{\text {th }}$ order corresponds to a wavelength

$$
\frac{\lambda}{n m}=2.60 \times 10^{-6}\left(\frac{x}{\text { pixel }}\right)^{2}+1.211 \times 10^{-2}\left(\frac{x}{\text { pixel }}\right)-0.137
$$

or taking the pixels size of $13.5 \mu \mathrm{m}$ into account we obtain for with the distance $x$ in $\mathrm{mm}$

$$
\frac{\lambda}{n m}=1.43 \times 10^{-2}\left(\frac{x}{\mathrm{~mm}}\right)^{2}+8.970 \times 10^{-1}\left(\frac{x}{\mathrm{~mm}}\right)-0.137 .
$$




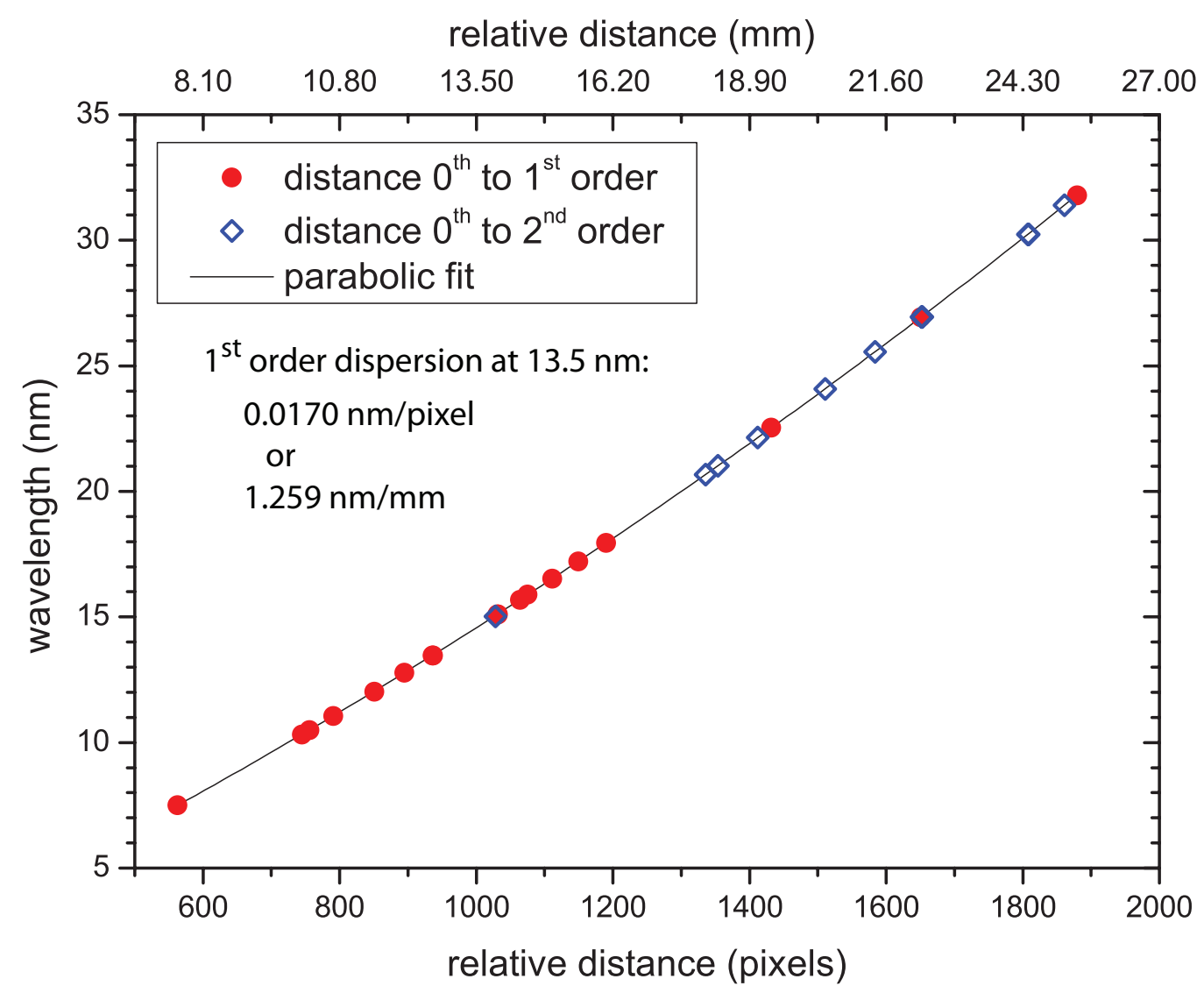

Figure 3. Measurement of the distance between different diffraction orders of the grating at various monochromator wavelength settings of the DORIS-III BW3 beam line. Data points from the $2^{\text {nd }}$ order have been entered with twice their wavelength to allow a common dispersion fit with the $1^{\text {st }}$ order.

In linear approximation, this corresponds to the above dispersion of $0.0170 \mathrm{~nm} / \operatorname{pixel}(1.259 \mathrm{~nm} / \mathrm{mm})$ at $\lambda=13.5 \mathrm{~nm}$. Generally, the dispersion can vary up to $15 \%$ for other source positions and grating angles than applied here. This measurement was conducted with the CCD located in the center of the flange and the $0^{\text {th }}$ order light focusing onto the $125^{\text {th }}$ pixel $(1.69 \mathrm{~mm})$ from the edge of the CCD. One should also point out that the linear fit dispersion for a given wavelength in first order will be slightly different from that in the second order of that wavelength. This is due to the non-linearity of the overall dispersion.

\subsection{Resolution}

To determine the resolution we use plasma line emission spectra from FEL and optical laser irradiated bulk aluminum (figure 4). In the case of optical laser irradiation, the lines have a natural width mainly determined by the plasma temperature. Therefore, the measured line width gives a lower limit for the resolution of the spectrograph where the plasma temperature is high, i.e. for the optical laser produced plasma. However, the FEL generates a relatively cold plasma $(\sim 34 \mathrm{eV}$ [5]) so that we can use those lines to estimate the spectrograph's resolution. They have an average width of $0.065 \mathrm{~nm}$ between $21 \mathrm{~nm}$ and $22 \mathrm{~nm}$. We also observe spectral structures from FEL single pulse scattering at $13.5 \mathrm{~nm}$ which have a width of $0.06 \mathrm{~nm}$. That agrees well with the FEL plasma 
lines at $21 \mathrm{~nm}$ and $22 \mathrm{~nm}$ and is evidence of the low dependance of the optics' imaging quality on the wavelength in this spectral region. From the average of the spectral widths we estimate that the spectrograph can resolve $\Delta \lambda=0.063 \pm 0.006 \mathrm{~nm}$ where the error is estimated using the standard deviation of the line widths. This corresponds to a resolution of $\lambda / \Delta \lambda=330_{-30}^{+35}$ at $21 \mathrm{~nm}$. The average line width for the optical laser generated plasma is $\Delta \lambda_{O L}=0.13 \pm 0.03 \mathrm{~nm}$, broader due to the higher plasma temperature. Figure 5 shows the sharpness of various emission lines in terms of $\lambda / \Delta \lambda$ for the optical laser and FEL generated plasma spectra from figure 4. Furthermore, we observed no significant change in resolution when rotating the grating. The dependance of the angle between focal plane and CCD on the grating rotation seems to be overcompensated by the large depth of field of the imaging system. An indication for the large depth of field is also that we observe no resolution change when varying the spectrograph source distance within approximately $\pm 1 \mathrm{~cm}$ around the optimum position.

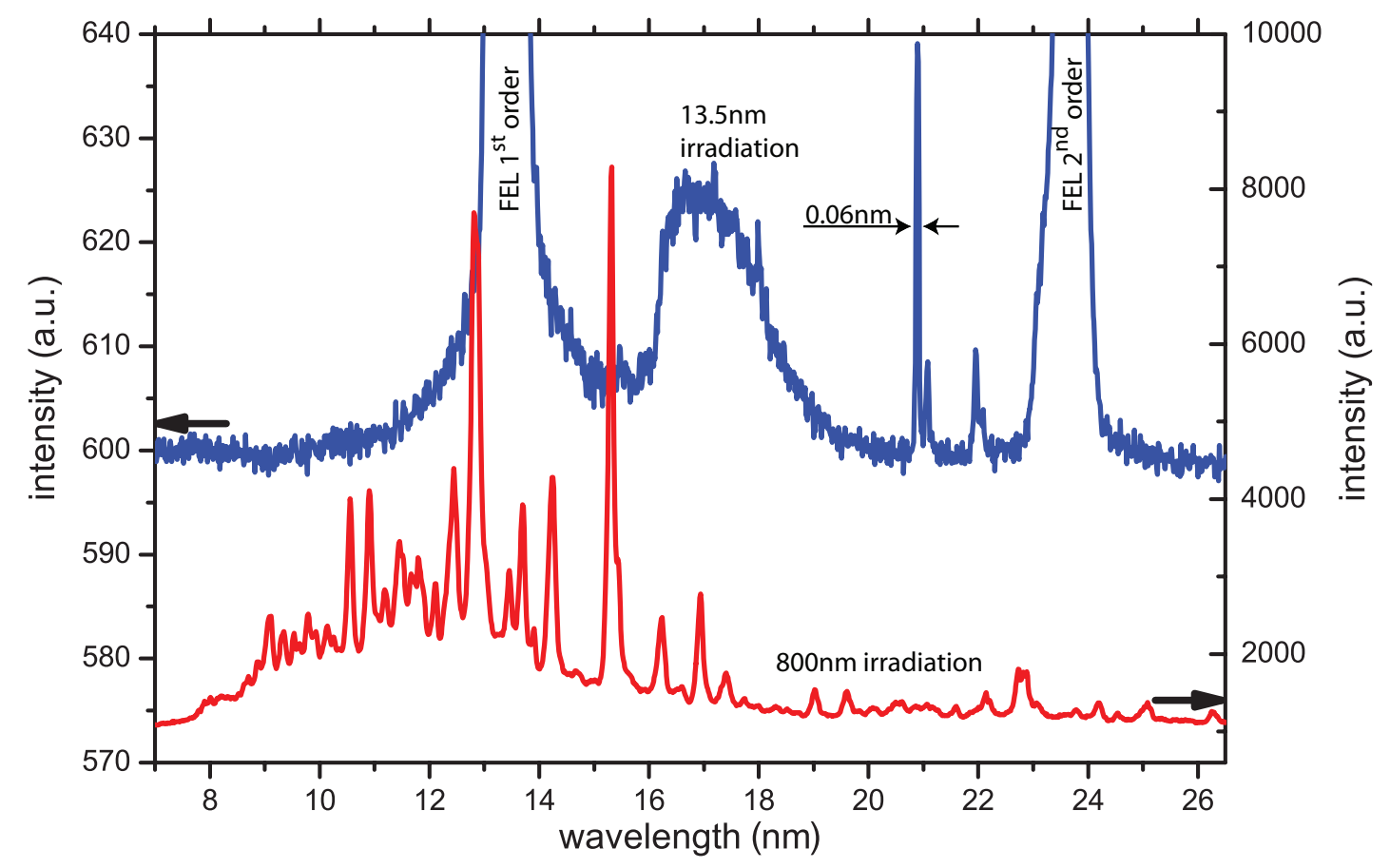

Figure 4. Comparison of soft X-ray emission spectra from bulk aluminium irradiated by $4 \times 10^{14} \mathrm{Wcm}^{-2}$ with a photon energy of $91.8 \mathrm{eV}\left(13.5 \mathrm{~nm}\right.$, without filter, top blue line) and $4 \times 10^{15} \mathrm{Wcm}^{-2}$ with a photon energy of $1.6 \mathrm{eV}(800 \mathrm{~nm}$, using a $200 \mathrm{~nm}$ zirconium-filter, bottom red line). From [13].

\subsection{Throughput}

Optimizing the spectrograph design for efficiency requires to maximize a figure of merit which is composed of the acceptance angle and the efficiency of the components. Here, we use the throughput $t(\lambda)$, defined by the wavelength-dependent ratio of photons, delivered to the detector, to isotropically emitted source photons. It is composed as

$$
t(\lambda)=\frac{\Omega}{4 \pi} \times m(\lambda) \times g_{1 s t}(\lambda)
$$




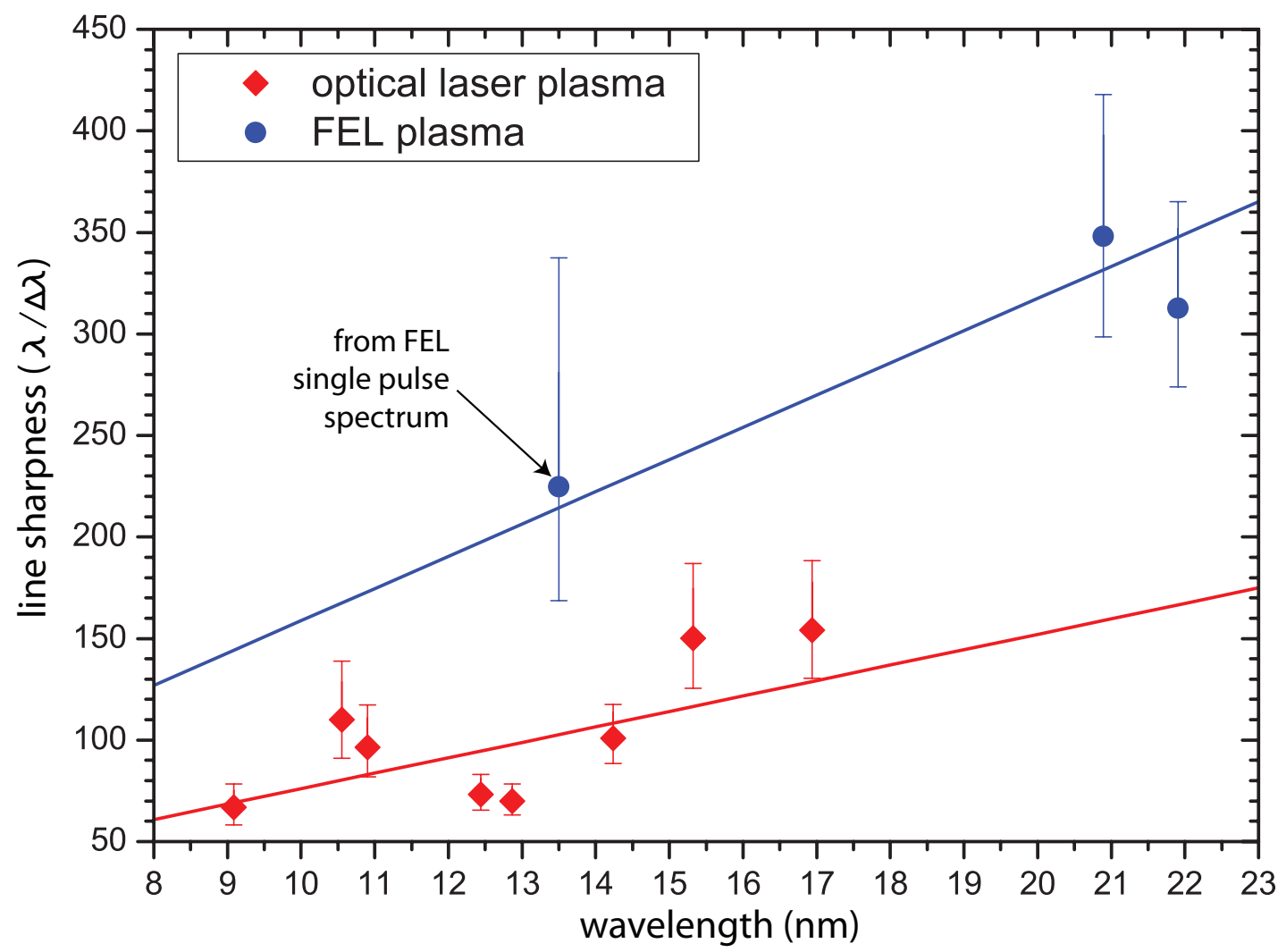

Figure 5. Sharpness $\lambda / \Delta \lambda$ of different optical laser (red diamonds) and FEL (blue circles) generated aluminium plasma lines. The sharpness of the plasma lines hardly deviates from the sharpness of their average plasma line widths, for the optical laser (red line, $\Delta \lambda_{O L}=0.13 \mathrm{~nm}$ ) and the FEL (blue line, $\Delta \lambda=0.063 \mathrm{~nm}$ ). This indicates stable imaging quality throughout the wavelength range.

with no filters used and where $\lambda$ is the wavelength, $\Omega$ the acceptance solid angle, $m(\lambda)$ the mirror reflectivity, and $g_{1 \text { st }}(\lambda)$ the gratings first order absolute efficiency. Table 1 compares this value for various types of spectrographs at $\lambda=13.5 \mathrm{~nm}$.

\begin{tabular}{|l|l|l|}
\hline Various spectrographs & $\Omega(\mathrm{sr})$ & $t(13.5 \mathrm{~nm})$ \\
\hline \hline HiTRaX (imaging toroidal mirror and VLS reflection grating) & $1.9 \times 10^{-3}$ & $2.9 \times 10^{-5}$ \\
\hline $\begin{array}{l}\text { Non-imaging spherical grating }\left(40 \times 100 \mathrm{~mm}^{2}, 5 \mathrm{~m} \text { radius) }\right. \\
350 \mathrm{~mm} \text { from source, Rowland geometry (similar [22, 23]) }\end{array}$ & $\sim 2 \times 10^{-3}$ & $\sim 2 \times 10^{-5}$ \\
\hline Non-imaging 1200 lines/mm VLS reflection grating [7, 8] & $1.2 \times 10^{-3}$ & $9.6 \times 10^{-6}$ \\
\hline Imaging toroidal mirror and transmission grating [9] & $4.1 \times 10^{-4}$ & $2.6 \times 10^{-6}$ \\
\hline $\begin{array}{l}\text { Pinhole }(100 \mu \mathrm{m}) \text { camera with 1500 lines/mm grating [20], } \\
\text { image distance } 20 \mathrm{~cm} \text {, and no magnification (e.g. [21]) }\end{array}$ & $2.0 \times 10^{-7}$ & $1.6 \times 10^{-9}$ \\
\hline
\end{tabular}

Table 1. Comparing different spectrographs for acceptance solid angle and throughput at $13.5 \mathrm{~nm}$ wavelength.

The wavelength-dependent efficiency of the spectrograph's components is shown in Figure 6. 
Each photon reaching the CCD generates $\sigma(\lambda)$ counts, where

$$
\sigma(\lambda)=\frac{h c}{\varepsilon \lambda} \times Q E(\lambda) \times A D C(\lambda)
$$

with the quantum efficiency $Q E(\lambda)$, the electron-hole-pair to count conversion $A D C(\lambda)$, Planck's constant $h$, and the speed of light $c$. The average energy to create an electron hole pair, $\varepsilon$, is $3.6 \mathrm{eV}$ for a typical silicon detector at room temperature. The quantum efficiency and the resulting countto-source photon ratio for different analog to digital converter (ADC) settings of the employed Princeton Instruments PIXIS-XO: $2 \mathrm{~KB}^{\mathrm{TM}}$ are also depicted in figure 6. ADC1, ADC2 and ADC3 correspond to $\mathrm{ADC}$ amplifications at the $2 \mathrm{MHz}$ low noise output where on average 3.56, 1.84 and 0.99 electrons produce one count, respectively. The vendor measured this for our particular camera. Thus, $\eta(\lambda)=t(\lambda) \times \sigma(\lambda)$ is the ratio of counts on the detector to isotropically emitted photons. Using $\eta(\lambda)$ the expected or observed signal in terms of counts on the CCD can be converted into the absolute number of isotropical source photons. A minimum amount of photons from the source is necessary to detect a signal. This detection threshold is determined by the signal-to-noise ratio $(S / N)$ of counts on the CCD. The root mean square noise on the CCD without illumination is 4.5 counts measured in a box of $50 \times 200$ pixel in the center of the CCD cooled to $\sim 200 \mathrm{~K}$ and along its major axis. Thus, the detection threshold for an minimum desirable $S / N=5$ is overcome with

$$
\frac{5 \times 4.5 \text { counts }}{9.2 \times 10^{-5} \text { counts } / \text { photons }}=2.4 \times 10^{5} \text { photons }
$$

emitted isotropically from a source smaller than the pixel size of the detector $\left(13.5 \times 13.5 \mu \mathrm{m}^{2}\right)$. For larger source sizes and non-imaging spectrographs the number of pixels over which the photons are spread needs to be taken into account. After the experiment, Figure 6 can be applied to determine the number of photons emitted from the source into $4 \pi$ solid angle and at a specific wavelength range. For example, this can be used to determine the free electron density in a plasma via the intensity of Bremsstrahlung emission [5].

\section{Integration into FLASH experiments}

The spectrograph was already successfully used in various experiments at FLASH [13, 15]. Due its small dimensions and weight we were able to attach it to vacuum chambers of various geometries. We achieved ultra high vacuum down to $10^{-8} \mathrm{mbar}$, allowing the simultaneous operation with pressure sensitive instruments like multi channel plates. The vacuum is also sufficiently clean to run a liquid hydrogen jet [13]. Furthermore, the stability of the spectrograph made changing from horizontal to vertical orientation seamless as the dispersion calibration was maintained. The integration into a DN $100 \mathrm{CF}$ manipulator made alignment to the source fast and saved valuable experimental time. The motorized positioning of grating and filter wheel has proven to work repeatable over many cycles. The plasma emission spectra (figure 4) with which we determined the resolution are examples of our experimental results.

\section{Conclusion}

In summary, the tests have shown that the High Throughput, High Resolution Spectrograph for Soft $\underline{X}$-Ray Light (HiTRaX) fulfills and even surpasses the design goals for Thomson Scattering 

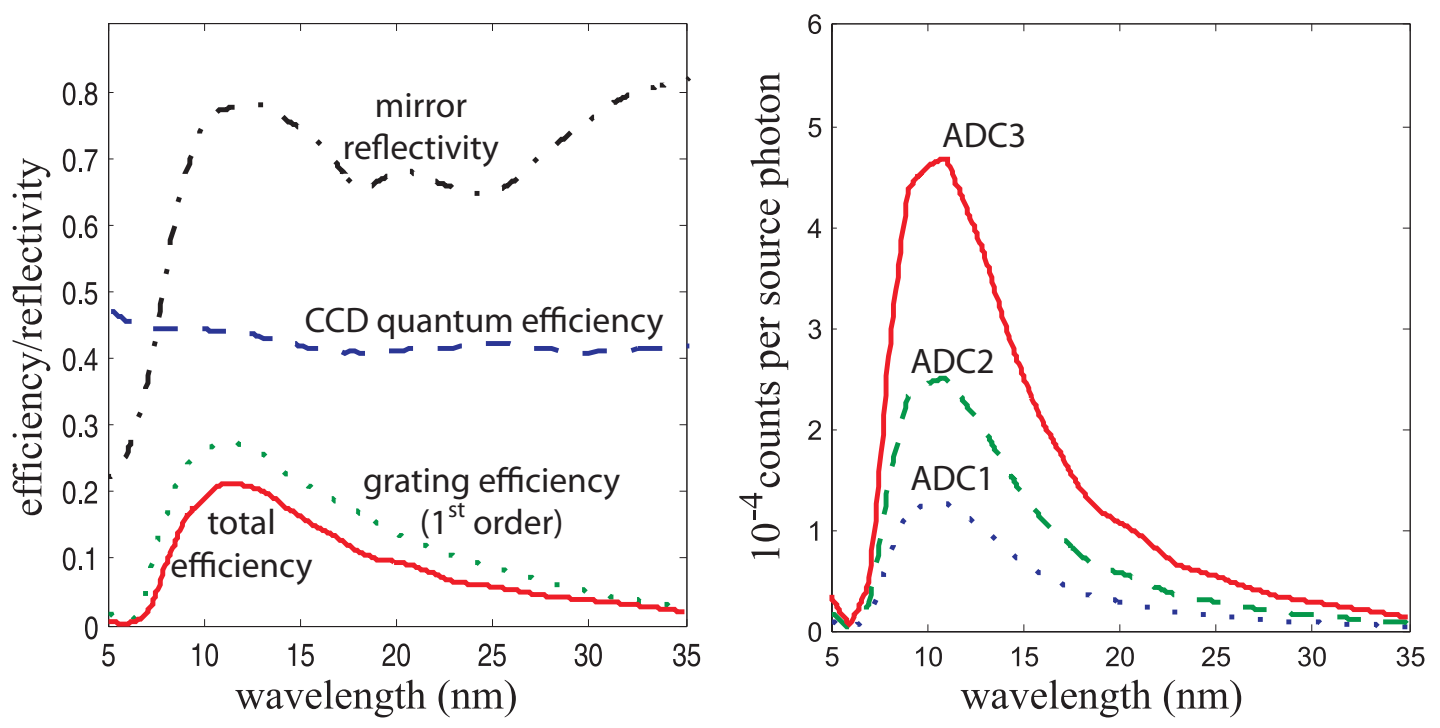

Figure 6. Efficiency or reflectivity of the spectrograph components according to the vendor specifications and the total efficiency of the optical elements (left), and the ratio of counts to isotropically emitted source photons (right) for different analog to digital converter (ADC) settings (see text).

plasma diagnostics at FLASH. The dispersion was calibrated and a resolution of $\lambda / \Delta \lambda=330$ measured at $21 \mathrm{~nm}$. A very high efficiency of the instrument was achieved, so that only $2.5 \times 10^{5}$ isotropic source photons at $13.5 \mathrm{~nm}$ yield the desirable signal to noise ratio of 5 . With this, the signal levels can be estimated before the experiment, and absolute photon numbers reconstructed from the experimental data.

In addition, low weight, the motorized grating and filter wheel rotation as well as the compact built within a DN $100 \mathrm{CF}$ manipulator make the spectrograph a versatile instrument and interesting for applications beyond Thomson Scattering. It has been successfully used for various FLASH experiments where its ease of use and integration into different setups have been practically shown. Emission spectroscopy experiments on materials excited with high intensity soft x-ray [15] or particle beams [24, 25] are also being conducted and planned for the future.

\section{Acknowledgments}

We thank the engineers L. Bittner and H. Lärz for their design effort and fruitful discussions as well as the mechanical workshops lead by J. Brehling, B. Klumbies and U. Mänz. We also thankfully acknowledge financial support by the Helmholtzgemeinschaft via the Virtual Institute VH-VI-104, the German Federal Ministry for Education and Research via Project No. FSP 301-FLASH. R.R.F. acknowledges DFG support under Grant No. GRK 1355.

\section{References}

[1] J. Nordgren et al., Rev. Sci. Instrum. 60, 1690 (1989).

[2] T. Ito and S. Okazaki, Nature 406, 1027 (2000).

[3] R. Giacconi et al., Space Sci. Rev. 9, 3 (1968). 
[4] A. C. Brinkman et al., Astrophys. J. Lett. 530, L111 (2000).

[5] U. Zastrau et al., Phys. Rev. E 78, 066406 (2008).

[6] T. Tschentscher and S. Toleikis, Eur. Phys. J. D 36, 193 (2005).

[7] T. Kita et al., Appl. Opt. 22, 512 (1983).

[8] N. Nakano et al., Appl. Opt. 23, 2386 (1984).

[9] J. Jasny et al., Rev. Sci. Instrum. 65, 1631 (1994).

[10] W. Ackermann et al., Nat. Photon. 1, 336 (2007).

[11] A. Höll et al., Euro. Phys. J. D 29, 159 (2004).

[12] A. Höll et al., High Energy Density Phys. 3, 120 (2007).

[13] S. Toleikis et al., High Energy Density Phys. 6, 15 (2010).

[14] S.H. Glenzer and R. Redmer, Rev. Mod. Phys. (2008). Accepted.

[15] B. Nagler et al., Nat. Phys. 5, 693 (2009).

[16] B. Henke, E. Gullikson, J. Davis, Atom. Data Nucl. Data 54, 181 (1993).

[17] A.R.B. de Castro and R. Reininger, Rev. Sci. Instrum. 63, 1317 (1992).

[18] C.U.S. Larsson et al., Nucl. Instrum. Methods A337, 603 (1994).

[19] R. Reininger and V. Saile, Nucl. Instrum. Methods A288, 343 (1989).

[20] H. W. Schnopper et al., Appl. Opt. 16, 1088 (1977).

[21] S. Sailaja et al., Meas. Sci. Technol. 9, 1462 (1998).

[22] J. Nordgren and R. Nyholm, Nucl. Instr. Meth. A 246, 242 (1986).

[23] S. Shin et al., Rev. Sci. Instrum. 66, 1584 (1995).

[24] D.H.H. Hoffmann et al., Laser Part. Beams 23, 47 (2005).

[25] P. K. Patel et al., Phys. Rev. Lett. 91, 125004 (2003). 